\title{
Editorial
}

\section{Acknowledgement to Reviewers of Pathogens in 2013}

\section{Pathogens Editorial Office}

MDPI AG, Klybeckstrasse 64, CH-4057 Basel, Switzerland

Published: 26 February 2014

The editors of Pathogens would like to express their sincere gratitude to the following reviewers for assessing manuscripts in 2013:

\begin{tabular}{lll} 
Allen, David J. & Charroux, Bernard & Grandadam, Marc \\
Andremont, Antoine & Cho, Sang-Nae & Gray, Wayne \\
Antonov, I. & Chu, Justin J. H. & Gubler, Duane \\
Atkinson, Robyn & Cialla, Dana & Guttman, Julian A. \\
Ayala-Del-Río, Héctor L. & Cohen, Marc & Haïk, Stéphane \\
Babu, Subash & Cordeiro, Yraima & Hao, Pei \\
Baltrus, David & D’auria, Giuseppe & Head, Mark \\
Barbet, Anthony & Dey, Bappaditya & Helmby, Helena \\
Baron, Thierry G. & Do, Thuy & Hershfield, Jeremy \\
Bessen, Richard & Donelli, Gianfranco & Holbrook, Michael \\
Biagi, Elena & Drebot, Michael & Hope, Jim \\
Bjarnsholt, Thomas & Fava, Francesca & Hurdle, Julian \\
Boone, David & Fernando, Samodha C. & Ike, K. \\
Bowman, John P. & Fischer, Wolfgang & Jesús, Requena \\
Boylan, J. & Fournier, Pierre-edouard & Jiménez-Ruiz, Elena \\
Bradford, B. J. & Gambetti, Pierluigi & Johnson, Reed F. \\
Brayton, Kelly A. & Ganta, Roman & Kemmerling, U. \\
Broder, Christopher & Gause, William C. & Kinchington, Paul \\
Buccigrossi, V. & Gillevet, Patrick & Knight, Rob \\
Budischak, Sarah & Glatzel, M. & Kosik-Bogacka, Danuta I. \\
Capozzoa, Alejandra Victoria & Gobet, Angélique & Kovacs, Gabor \\
Castilla, Joaquin & Goldsmith, Jason & Labruyere, Elisabeth \\
Catry, Boudewijn & Gomes, Joana & Larsen, Lone Heimann \\
Cavalieri, Francesca & Gomez-Llorente, Carolina & Lass-Flörl, C. \\
\hline & &
\end{tabular}




\begin{tabular}{lll} 
Lo, Michael & Lee, Hyoung-gon & Seuberlich, Torsten \\
Lulich, Jody & Lin, Jin-Ming & Sly, Laura \\
Maeda, Shin & Othman, Ahmad & Snyder, Chris \\
Maresso, Anthony & Pagano, Joseph S. & Sonawane, Avinash \\
Martinez-Sobrido, Luis & Peek, Richard M. & Sørensen, Søren Johannes \\
Martinez, Jose Luis & Petri, Daniel & Stack, Mick \\
Mathey-Prevot, Bernard & Pillar, Dylan & Sukumaran, Bindu \\
Matthias, Michael A. & Prabha, Vijay & Tang, Chuan Y. \\
McDonald, L. Clifford & Reichel, Michael & Tsou, Lun \\
McLean, Catriona & Reimer, Aleisha & Van Beek, J. \\
Mcmurray, David N. & Rejmanek, Daniel & Vilette, Didier \\
Mehaffy, Carolina & Reljic, Rajko & Walker, David \\
Midtvedt, Tore & Rey, Federico & Walter, Jens \\
Minton, J. Ernest & Rockx, Barry & Wang, Dan \\
Mizoguchi, Atsushi & Röhrl, Johann & Wang, Fei \\
Mollapour, Mehdi & Romalde, Jesús López & Warawa, Jonathan \\
Montagne, Lucile & Rubenstein, Richard & Whitehouse, Michael \\
Moore, Ben & Rǒžek, Daniel & Williams, Dustin L. \\
Moore, D. P. & Sarris, Panagiotis F. & Wong, K. T. \\
Moreno, J. J. & Schares, Gereon & Yang, Liang \\
Murray, Paul G. & Schaub, Günter A. & Yeh, Yi-Chun \\
Musch, Mark & Schaudies, R. Paul & Yin, Hang \\
Nava, Porfirio & Schmid-Hempel, Paul & Yokoyama, Takashi \\
Nishida, Noriyuki & Scorpio, Diana G. & Yu, Xuejie \\
Niyonsaba, François & Scortichini, Marco & Zerr, Inga \\
Oliver, W. T. & Serra, A. & Zilbauer, Matthias \\
Lauber, Christian L. & Serrano-Luna, Jesús & \\
& & \\
\hline & &
\end{tabular}

(C) 2014 by the authors; licensee MDPI, Basel, Switzerland. This article is an open access article distributed under the terms and conditions of the Creative Commons Attribution license (http://creativecommons.org/licenses/by/3.0/). 